\title{
Analysis of Pharmacist Interventions Used to Resolve Safety Target of Polypharmacy (STOP) Drug Interactions
}

\author{
Barbara Kasper, PharmD, BCACP; Angela Erdel, PharmD, BCPS; Caitlynn Tabaka, PharmD; and Borden Edgar, PharmD
}

Background: Statin drug interactions commonly increase the risk of muscle-related toxicities. The medical literature supports consultative pharmacist interventions to resolve drug interactions, but studies demonstrating autonomous pharmacist interventions are lacking.

Objective: To evaluate the complementary impact of using pharmacist-led protocols and pharmacists with prescriptive authority to resolve statin drug interactions.

Methods: Pharmacist-led protocols were developed to address gemfibrozil-statin and niacin-statin interactions. Pharmacists discontinued gemfibrozil and niacin by protocol or referred patients to the Patient Aligned Care Team (PACT) Pharmacy Clinic for individualized management. After all drug interactions were addressed, a retrospective quality improvement analysis was conducted. The primary outcome was to evaluate the impact of gemfibrozil and niacin discontinuation by protocol on patients' triglyceride (TG) laboratory results. The coprimary endpoints were the change in TGs and the percentage of patients with TGs $\geq 500 \mathrm{mg} / \mathrm{dL}$, following pharmacist discontinuation by protocol. Secondary outcomes included the time required to resolve the interactions and a description of the PACT Clinical Pharmacy Specialists' (CPS) pharmacologic interventions.
Results: The gemfibrozil and niacin protocols addressed 397 drug interactions. Seventy-six patients underwent gemfibrozil discontinuation by protocol and had TG laboratory results available. TG levels decreased or increased by $<100 \mathrm{mg} / \mathrm{dL}$ for 62 patients (82\%), and 1 patient (1.3\%) experienced TG elevation above the threshold of $500 \mathrm{mg} / \mathrm{dL}$. Thirty-six patients had niacin discontinued by protocol and available laboratory results. The TG levels decreased or increased by $<100 \mathrm{mg} / \mathrm{dL}$ for 33 patients (91.7\%), and no patients had TG levels increase above the threshold of $500 \mathrm{mg} / \mathrm{dL}$. The mean time required to resolve both gemfibrozil and niacin drug interactions was 15.5 minutes per patient. A total of 129 patients were referred to the PACT Pharmacy Clinic to manage gemfibrozil and niacin drug interactions. TG laboratory results were available for 80 gemfibrozil patients (74.8\%) and 16 niacin patients (72.7\%). The PACT CPS made 171 pharmacologic interventions to address drug interactions and the median of 2 encounters per patient.

Conclusions: This single-site quality improvement analysis supports the complementary use of protocols and pharmacists with prescriptive authority to resolve statin drug interactions. These data support expanded roles for pharmacists, across settings, to mitigate select drug interactions.
Barbara Kasper is a Clinical Assistant Professor; and Borden Edgar and Caitlynn Tabaka were Students at the time this article was written; all at the University of Missouri-Kansas City School of Pharmacy. Angela Erdel and Barbara Kasper are Clinical Pharmacy Specialists at the Harry S. Truman Memorial Veterans' Hospital in Columbia, Missouri. Correspondence: Barbara Kasper (kasperb@umkc.edu)
$(0$ tatins are one of the most common medications dispensed in the US and are associated with clinically significant drug interactions. ${ }^{1,2}$ The most common adverse drug reaction (ADR) of statin drug interactions is muscle-related toxicities. ${ }^{2}$ Despite technology advances to alert clinicians to drug interactions, updated statin manufacturer labeling, and guideline recommendations, inappropriate prescribing and dispensing of statin drug interactions continues to occur in health care systems. ${ }^{2-10}$

The medical literature has demonstrated many opportunities for pharmacists to prevent and mitigate drug interactions. At the points of prescribing and dispensing, pharmacists can reduce the number of potential drug interactions for the patient. ${ }^{11-13}$ Pharmacists also have identified and resolved drug interactions through quality assurance review after dispensing to a patient. ${ }^{7,8}$

Regardless of the time point of an intervention, the most common method pharmacists used to resolve drug interactions was through recommendations to a prescriber. The recommendations were generated through academic detailing, clinical decision support algorithms, drug conversions, or the pharmacist's expertise. Regardless of the method the pharmacist used, the prescriber had the final authority to accept or decline the recommendation. ${ }^{7,8,11-13} \mathrm{Al}$ though these interventions were effective, pharmacists could further streamline the process by autonomously resolving drug interactions. However, these types of interventions are not well described in the medical literature.

\section{BACKGROUND}

The US Department of Veterans Affairs (VA) Veterans Integrated Service Network (VISN), established the Safety Target of Polypharmacy (STOP) report in 2015. At each facility in the network, the report identified patients who were dispensed medications known to have drug interactions. The interactions were chosen by the 
VISN, and the severity of the interactions was based on coding parameters within the VA computerized order entry system, which uses a severity score based on First Databank data. At the Harry S. Truman Memorial Veterans' Hospital (Truman VA) in Columbia, Missouri, > 500 drug interactions were initially active on the STOP report. The most common drug interactions were statins with gemfibrozil and statins with niacin. ${ }^{14-18}$ The Truman VA Pharmacy Service was charged with resolving the interactions for the facility.

The Truman VA employs 3 Patient Aligned Care Team (PACT) Clinical Pharmacy Specialists (CPS) practicing within primary care clinics. PACT is the patientcentered medical home model used by the VA. PACT CPS are ambulatory care pharmacists who assist providers in managing diseases using a scope of practice. Having a scope of practice would have allowed the PACT CPS to manage drug interactions with independent prescribing authority. However, due to the high volume of STOP report interactions and limited PACT CPS resources, the Pharmacy Service needed to develop an efficient, patient-centered method to resolve them. The intervention also needed to allow pharmacists, both with and without a scope of practice, to address the interactions.

\section{METHODS}

The Truman VA Pharmacy Service developed protocols, approved by the Pharmacy and Therapeutics (P\&T) Committee, to manage the specific gemfibrozil-statin and niacinstatin interactions chosen for the VISN 15 STOP report (Figures 1 and 2). The protocols were designed to identify patients who did not have a clear indication for gemfibrozil or niacin, were likely to maintain triglycerides (TGs) $<500 \mathrm{mg} / \mathrm{dL}$ without these medications, and would not likely require close monitoring after discontinuation. ${ }^{19}$ The protocols allowed pharmacists to autonomously discontinue gemfibrozil or niacin if patients did not have a history of pancreatitis, TGs $\geq 400 \mathrm{mg} / \mathrm{dL}$ or a nonlipid indication for niacin (eg, pellagra) after establishing care at Truman VA. Additionally, both interacting medications had to be dispensed by the VA. When pharmacists discontinued a medica-
FIGURE 1 Gemfibrozil Protocol

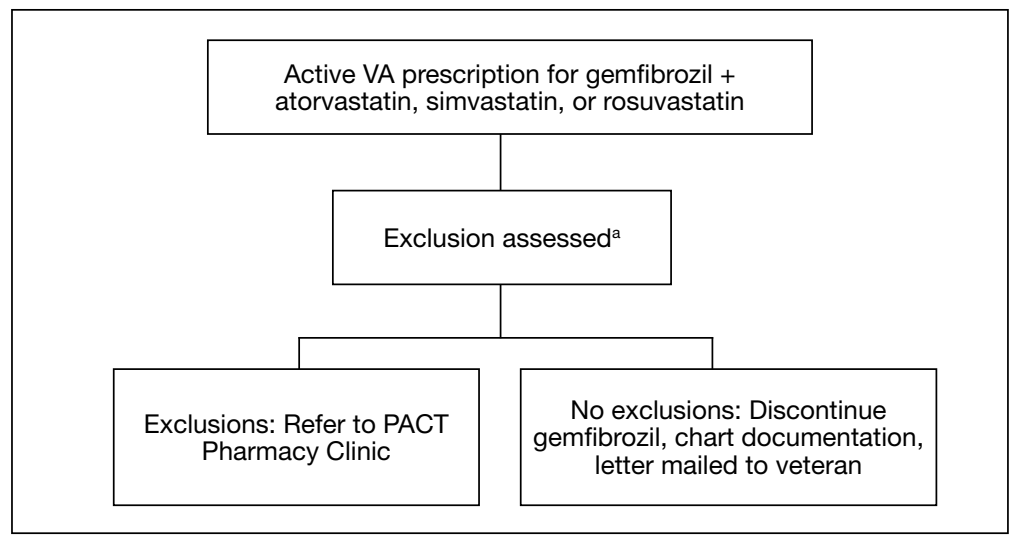

Abbreviations: PACT, Patient Aligned Care Team; VA, US Department of Veterans Affairs. aTriglycerides $\geq 400 \mathrm{mg} / \mathrm{dL}$, history of pancreatitis, non-VA statin and/or gemfibrozil.

\section{FIGURE 2 Niacin Protocol}

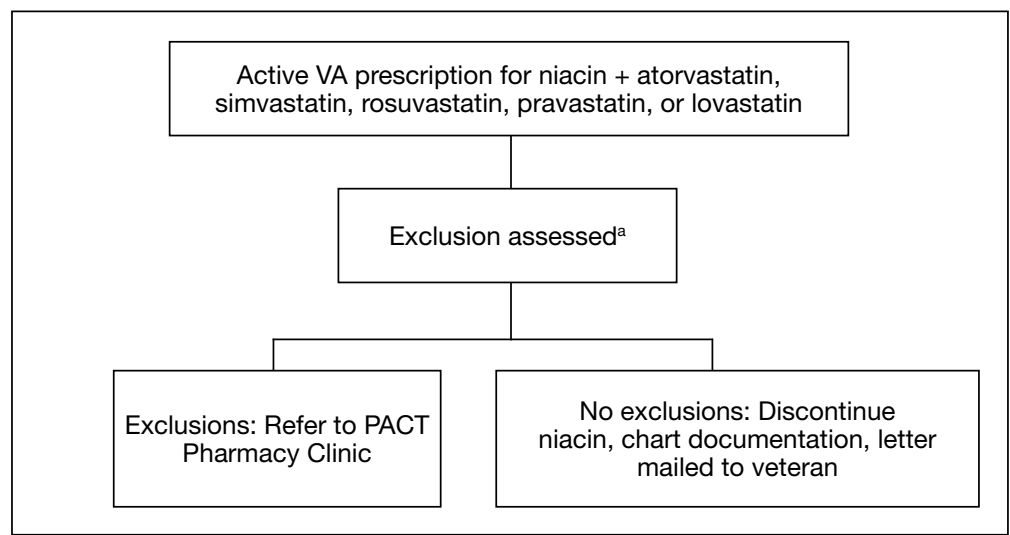

Abbreviations: PACT, Patient Aligned Care Team; VA, US Department of Veterans Affairs. aTriglycerides $\geq 400 \mathrm{mg} / \mathrm{dL}$, history of pancreatitis due to elevated triglycerides, non-VA statin and/or niacin, nonlipid indication for niacin (pellagra).

tion, it was documented in a note in the patient electronic health record. The prescriber was notified through the note and the patient received a notification letter. Follow-up laboratory monitoring was not required as part of the protocol.

If patients met any of the exclusion criteria for discontinuation, the primary care provider (PCP) was notified to place a consult to the PACT Pharmacy Clinic for individualized interventions and close monitoring. Patients prescribed niacin for nonlipid indications were allowed to continue with their current drug regimen. At each encounter, the PACT CPS assessed for ADRs, made individualized medication changes, and arranged follow-up appointments. Once the interaction was resolved and treatment 
TABLE 1 Patients Receiving Interacting Drugs Identified by STOP Reports

\begin{tabular}{lcc} 
Baseline Characteristics & $\begin{array}{c}\text { Gemfibrozil, No. (\%) } \\
(\mathbf{n}=\mathbf{3 1 7})\end{array}$ & $\begin{array}{c}\text { Niacin, No. (\%) } \\
\mathbf{( n = 8 0 )}\end{array}$ \\
\hline Age groups, y & & \\
$20-39$ & $3(0.9)$ & $0(0)$ \\
$40-59$ & $62(19.6)$ & $10(12.5)$ \\
$60-79$ & $225(71)$ & $59(73.8)$ \\
$80-99$ & $27(8.5)$ & $11(13.8)$ \\
\hline Race & & \\
African American & $4(1.3)$ & $1(1.3)$ \\
American Indian, Alaska Native & $6(1.9)$ & $2(2.5)$ \\
Asian & $0(0)$ & $1(1.3)$ \\
White & $306(96.5)$ & $76(95)$ \\
Native Hawaiian, Pacific Islander & $1(0.3)$ & $0(0)$ \\
\hline Gender, male & $311(98.1)$ & $80(100)$ \\
\hline Interacting medications & & \\
Atorvastatin & $128(40.4)$ & $31(38.8)$ \\
Lovastatin & $0(0)$ & $2(2.5)$ \\
Pravastatin & $0(0)$ & $6(7.5)$ \\
Rosuvastatin & $66(20.8)$ & $19(23.8)$ \\
Simvastatin & $123(38.8)$ & $22(27.5)$ \\
Abbreviation: STOP, Safety Target of Polypharmacy. & &
\end{tabular}

FIGURE 3 Patient Disposition Following Gemfibrozil Protocol

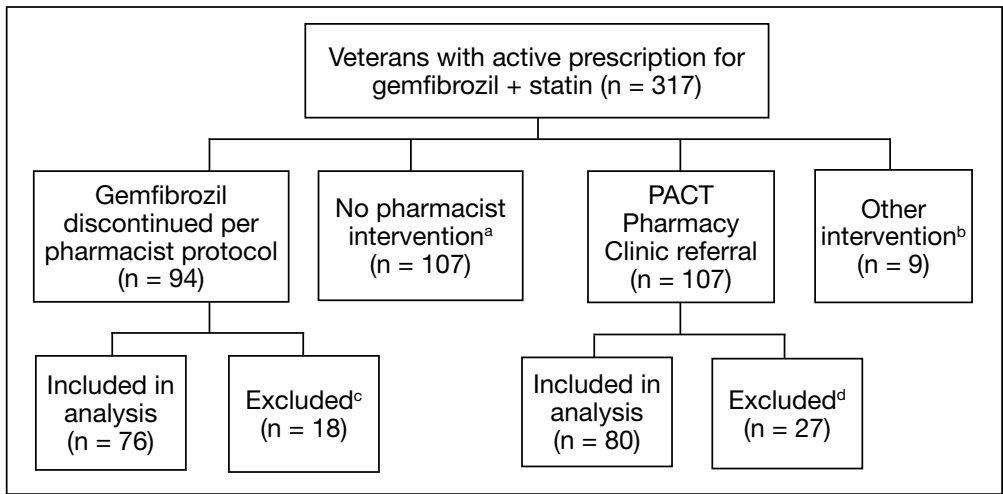

Abbreviation: PACT, Patient Aligned Care Team.

alnteraction was resolved prior to pharmacist review, $\geq 1$ interacting medications was expired and not continued, patient self-discontinued $\geq 1$ interacting medications, patient deceased, patient moved, patient receiving $\geq 1$ interacting medications outside the VA.

'Other intervention: prescriber resolved interaction following pharmacist notification outside of algorithm.

"Pharmacist did not follow protocol, missing follow-up laboratory results.

dNonpharmacist provider made intervention(s) while enrolled in PACT Pharmacy Clinic or resumed management before patient completed all PACT Pharmacy Clinic follow-up, PACT CPS unable to make initial contact with patient, or missing follow-up laboratory results.

goals met, the PCP resumed monitoring of the patient's lipid therapy.

Following all pharmacist interventions, a retrospective quality improvement analysis was conducted. The primary outcome was to evaluate the impact of discontinuing gemfibrozil and niacin by protocol on patients' laboratory results. The coprimary endpoints were to describe the change in TG levels and the percentage of patients with TGs $\geq 500 \mathrm{mg} / \mathrm{dL}$ at least 5 weeks following the pharmacist-directed discontinuation by protocol. Secondary outcomes included the time required to resolve the interactions and a description of the PACT CPS pharmacologic interventions. Additionally, a quality assurance peer review was used to ensure the pharmacists appropriately utilized the protocols.

Data were collected from August 2016 to September 2017 for patients prescribed gemfibrozil and from May 2017 to January 2018 for patients prescribed niacin. The time spent resolving interactions was quantified based on encounter data. Descriptive statistics were used to analyze demographic information and the endpoints associated with each outcome. The project was reviewed by the University of Missouri Institutional Review Board, Truman VA privacy and information security officers, and was determined to meet guidelines for quality improvement.

\section{RESULTS}

The original STOP report included 397 drug interactions involving statins with gemfibrozil or niacin (Table 1). The majority of patients were white and male aged 60 to 79 years. Gemfibrozil was the most common drug involved in all interactions (79.8\%). The most common statins were atorvastatin (40\%) and simvastatin (36.5\%).

\section{Gemfibrozil-Statin Interactions}

Pharmacists discontinued gemfibrozil by protocol for 94 patients (29.6\%), and 107 patients $(33.8 \%)$ were referred to the PACT Pharmacy Clinic (Figure 3). For the remaining 116 patients (36.6\%), the drug interaction was addressed outside of the protocol for the following reasons: the drug interaction was resolved prior to pharmacist review; an interacting prescription was expired and not to be continued; the patient self-discontinued $\geq 1$ interacting medications; the patient was deceased; the patient moved; the patient was receiving $\geq 1$ interacting medications outside of the VA; or the prescriber resolved the interaction following notification by the pharmacist. 
Ultimately, the interaction was resolved for all patients with a gemfibrozil-statin interaction on the STOP report. Following gemfibrozil discontinuation by protocol, 76 patients (80.9\%) had TG laboratory results available and were included in the analysis. Sixty-two patients' (82\%) TG levels decreased or increased by $<100 \mathrm{mg} / \mathrm{dL}$ (Figure 4), and the TG levels of 1 patient (1.3\%) increased above the threshold of $500 \mathrm{mg} / \mathrm{dL}$. The mean (SD) time to the first laboratory result after the pharmacists mailed the notification letter was 6.5 (3.6) months (range, 1-17). The pharmacists spent a mean of 16 minutes per patient resolving each interaction.

Of the 107 patients referred to the PACT Pharmacy Clinic, 80 (74.8\%) had TG laboratory results available and were included in the analysis. These patients were followed by the PACT CPS until the drug interaction was resolved and confirmed to have TG levels at goal $(<500 \mathrm{mg} / \mathrm{dL})$. Gemfibrozil doses ranged from $300 \mathrm{mg}$ daily to $600 \mathrm{mg}$ twice daily, with $70 \%(\mathrm{n}=56)$ of patients taking $600 \mathrm{mg}$ twice daily. The PACT CPS made 148 interventions (Table $2)$. Twenty-three (29\%) patients required only gemfibrozil discontinuation. The remaining 57 patients $(71 \%)$ required at least 2 medication interventions. The PACT CPS generated 213 encounters for resolving drug interactions with a median of 2 encounters per patient.

Quality assurance review identified 5 patients (5.3\%) who underwent gemfibrozil discontinuation by protocol, despite having criteria that would have recommended against discontinuation. In accordance with the protocol criteria, these patients were later referred to the PACT Pharmacy Clinic. None of these patients experienced a TG increase at or above the threshold of $500 \mathrm{mg} / \mathrm{dL}$ after gemfibrozil was initially discontinued but were excluded from the earlier analysis.

\section{Niacin-Statin Interactions}

Pharmacists discontinued niacin by protocol for 48 patients $(60.0 \%)$, and 22 patients (27.5\%) were referred to the PACT Pharmacy Clinic (Figure 5). For the remaining 5 patients $(6.3 \%)$, the interaction was either addressed outside the protocol prior to pharmacist review, or an interacting pre-
TABLE 2 PACT Pharmacy Clinic Interventions Used to Manage Gemfibrozil/Niacin-Statin Interactions

\begin{tabular}{lc}
\hline Interventions & No. (\%) \\
\hline Gemfibrozil-statin interactions $(\mathrm{n}=80)$ & $23(29)$ \\
Discontinue gemfibrozil & $5(6)$ \\
Discontinue gemfibrozil and other lipid medication ${ }^{\mathrm{a}}$ & $4(5)$ \\
Discontinue gemfibrozil and decrease statin & $25(31)$ \\
Discontinue gemfibrozil and increase statin & $9(11)$ \\
Discontinue gemfibrozil and other lipid medication, increase statin & \\
Discontinue gemfibrozil and start fish oil & $2(3)$ \\
Discontinue gemfibrozil, increase statin, and start fish oil & $3(4)$ \\
Discontinue gemfibrozil and convert statin & $4(5)$ \\
Discontinue gemfibrozil, convert statin, and start fish oil & $1(1)$ \\
Discontinue statin & $2(3)$ \\
Discontinue statin and increase gemfibrozil & $1(1)$ \\
Discontinue statin and start fish oil & $1(1)$ \\
\hline Niacin-statin interactions (n = 16) & $12(75)$ \\
Discontinue niacin & $3(19)$ \\
Discontinue niacin and simvastatin, start atorvastatin & $1(6)$ \\
Discontinue niacin and increase rosuvastatin &
\end{tabular}

afish oil, niacin, ezetimibe, cholestyramine, and/or colestipol.

scription was expired and not to be continued. Additionally, niacin was continued per prescriber preference in 5 patients (6.3\%).

Thirty-six patients (75\%) had TG laboratory results available following niacin discontinuation by protocol and were included in the analysis. Most patients' ( $\mathrm{n}=$ 33, 91.7\%) TG levels decreased or increased by $<100 \mathrm{mg} / \mathrm{dL}$. No patient had a TG level that increased higher than the threshold of $500 \mathrm{mg} / \mathrm{dL}$. The mean (SD) time to the first laboratory result after the pharmacists mailed the notification letter, was 5.3 (2.5) months (range, 1.2-9.8). The pharmacists spent a mean of 15 minutes per patient resolving each interaction. The quality assurance review found no discrepancies in the pharmacists' application of the protocol.

Of the 22 patients referred to the PACT Pharmacy Clinic, 16 (72.7\%) patients had TG laboratory results available and were included in the analysis. As with the gemfibrozil interactions, these patients were followed by the PACT Pharmacy Clinic until the drug interaction was resolved and confirmed to have TGs at goal $(<500 \mathrm{mg} / \mathrm{dL})$. Niacin doses ranged from $500 \mathrm{mg}$ daily to 2,000 $\mathrm{mg}$ daily, with the majority of patients taking $1,000 \mathrm{mg}$ daily. The PACT CPS made 23 interventions. The PACT CPS generated 46 encounters for resolving drug interactions with a median of 2 encounters per patient. 


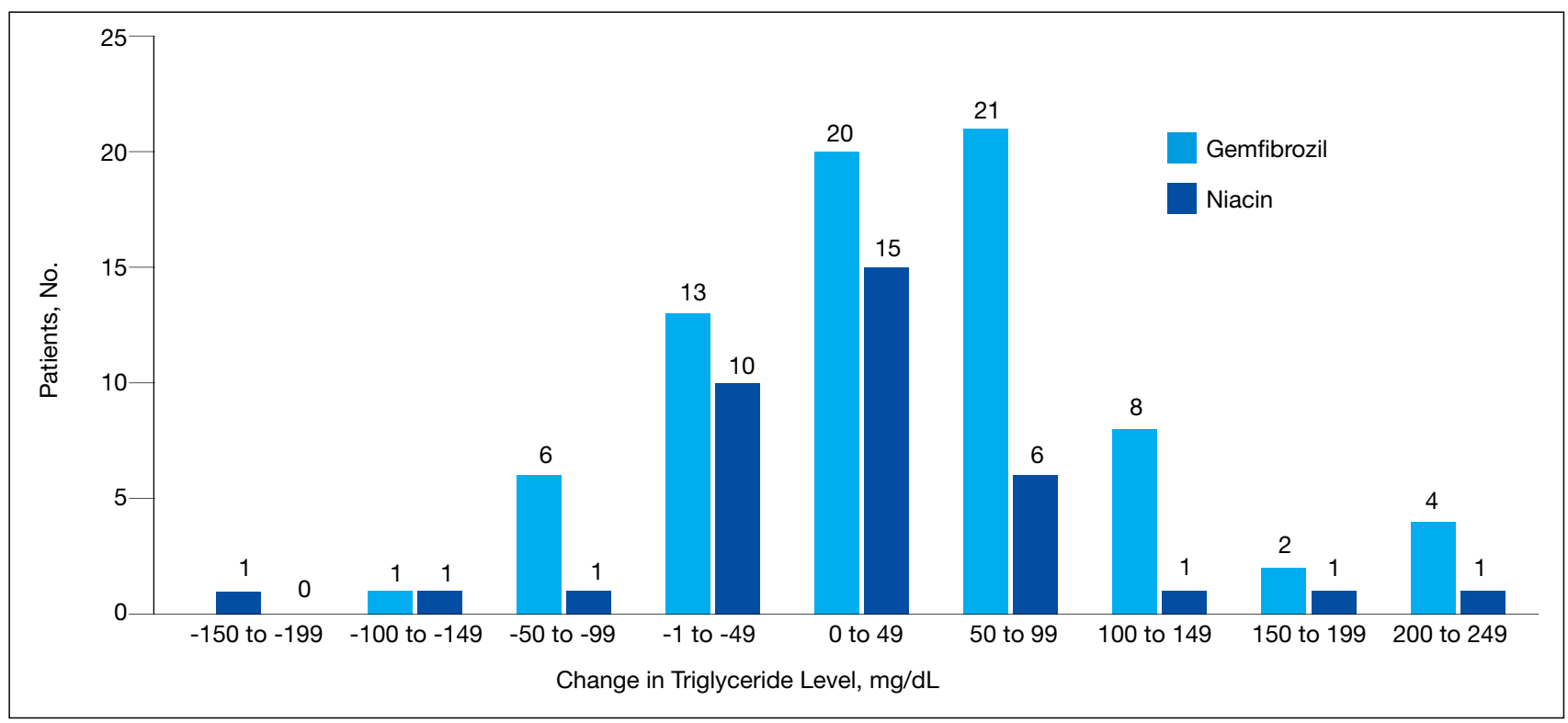

\section{DISCUSSION}

Following gemfibrozil or niacin discontinuation by protocol, most patients with available laboratory results experienced either a decrease or modest TG elevation. The proportion of patients experiencing a decrease in TGs was unexpected but potentially multifactorial. Individual causes for the decrease in TGs were beyond the scope of this analysis. The retrospective design limited the ability to identify variables that could have impacted TG levels when gemfibrozil or niacin were started and discontinued. Although the treatment of TG levels is not indicated until it is $\geq 500 \mathrm{mg} / \mathrm{dL}$, due to an increased risk of pancreatitis, both protocols excluded patients with a history of TGs $\geq 400 \mathrm{mg} / \mathrm{dL} .{ }^{19}$ The lower threshold was set to compensate for anticipated increase in TG levels, following gemfibrozil or niacin discontinuation, and to minimize the number of patients with TG levels $\geq 500 \mathrm{mg} / \mathrm{dL}$. The actual impact on patients' TG levels supports the use of this lower threshold in the protocol.

When TG levels increased by 200 to $249 \mathrm{mg} / \mathrm{dL}$ after gemfibrozil or niacin discontinuation, patients were evaluated for possible underlying causes, which occurred for 4 gemfibrozil and 1 niacin patient. One patient started a $\beta$-blocker after gemfibrozil was initiated, and 3 patients were tak- ing gemfibrozil prior to establishing care at the VA. The TG levels of the patient taking niacin correlated with an increased hemoglobin $A_{1 c}$. The TG level for only 1 patient taking gemfibrozil increased above the 500 $\mathrm{mg} / \mathrm{dL}$ threshold. The patient had several comorbidities known to increase TG levels, but the comorbidities were previously well controlled. No additional medication changes were made at that time, and the TG levels on the next fasting lipid panel decreased to goal. The patient did not experience any negative clinical sequelae from the elevated TG levels.

Thirty-five patients (36\%) who were referred to the PACT Pharmacy Clinic required only either gemfibrozil or niacin discontinuation. These patients were evaluated to identify whether adjustments to the protocols would have allowed for pharmacist discontinuation without referral to the PACT Pharmacy Clinic. Twenty-four of these patients (69\%) had repeated TG levels $\geq 400 \mathrm{mg} / \mathrm{dL}$ prior to referral to the PACT Pharmacy Clinic. Additionally, there was no correlation between the gemfibrozil or niacin doses and the change in TG levels following discontinuation. These data indicate the protocols appropriately identified patients who did not have an indication for gemfibrozil or niacin. 
In addition to drug interactions identified on the STOP report, the PACT CPS resolved 12 additional interactions involving simvastatin and gemfibrozil. Additionally, unnecessary lipid medications were deprescribed. The PACT CPS identified 13 patients who experienced myalgias, an ADR attributed to the gemfibrozil-statin interaction. Of those, 9 patients' ADRs resolved after discontinuing gemfibrozil alone. For the remaining 4 patients, additional interventions to convert the patient to another statin were required to resolve the ADR.

Using pharmacists to address the drug interactions shifted workload from the prescribers and other primary care team members. The mean time spent to resolve both gemfibrozil and niacin interactions by protocol was 15.5 minutes. One hundred fortytwo patients (35.8\%) had drug interactions resolved by protocol, saving the PACT CPS' expertise for patients requiring individualized interventions. Drug interactions were resolved within 4 PACT CPS encounters for $93.8 \%$ of the patients taking gemfibrozil and within 3 PACT CPS encounters for $93.8 \%$ of the patients taking niacin.

The protocols allowed 12 additional pharmacists who did not have an ambulatory care scope of practice to assist the PACT CPS in mitigating the STOP drug interactions. These pharmacists otherwise would have been limited to making consultative recommendations. Simultaneously, the design allowed for the PACT pharmacists' expertise to be allocated for patients most likely to require interventions beyond the protocols. This type of intraprofessional referral process is not well described in the medical literature. To the authors' knowledge, the only studies described referrals from hospital pharmacists to community pharmacists during transitions of care on hospital discharge. ${ }^{20,21}$

\section{Limitations}

The results of this study are derived from a retrospective chart review at a single VA facility. The autonomous nature of PACT CPS interventions may be difficult to replicate in other settings that do not permit pharmacists the same prescriptive authority. This analysis was designed to demonstrate the
Figure 5 Patient Disposition Following Niacin Protocol

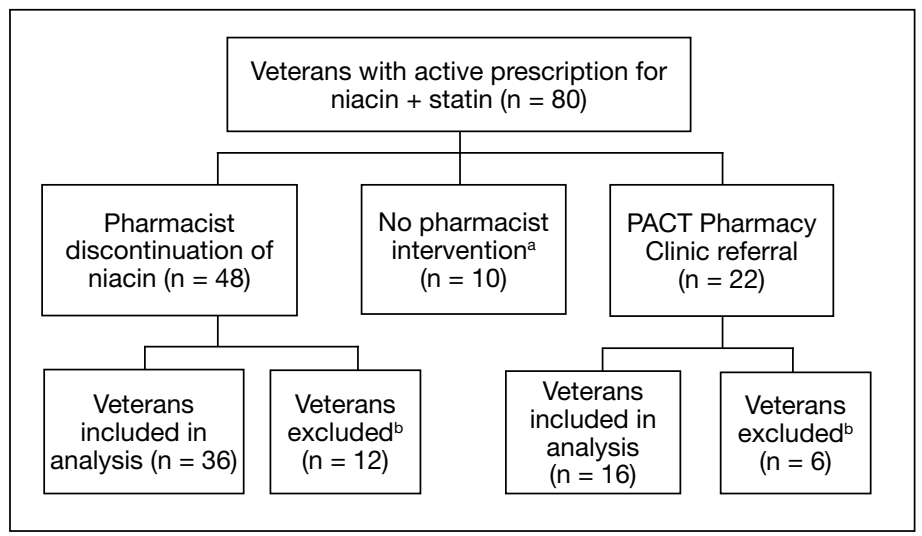

Abbreviation: PACT, Patient Aligned Care Team.

aPrescriber elected to continue interacting combination of medications

$(n=5)$, interaction was resolved prior to pharmacist review $(n=4)$, or $\geq 1$ interacting medications were expired and not to be continued $(n=1)$.

bExcluded due to missing follow-up laboratory results.

impact of the pharmacist in resolving major drug interactions. Patients referred to the PACT Pharmacy Clinic who also had their lipid medications adjusted by a nonpharmacist provider were excluded. However, this may have minimized the impact of the PACT CPS on the patient care provided. As postintervention laboratory results were not available for all patients, some patients' TG levels could have increased above the $500 \mathrm{mg} / \mathrm{dL}$ threshold but were not identified. The time investment was extensive and likely underestimates the true cost of implementing the interventions.

Because notification letters were used to instruct patients to stop gemfibrozil or niacin, several considerations need to be addressed when interpreting the follow-up laboratory results. First, we cannot confirm whether the patients received the letter or the exact date the letter was received. Additionally, we cannot confirm whether the patients followed the instructions to stop the interacting medications or the date the medications were stopped. It is possible some patients were still taking the interacting medication when the first laboratory was drawn. Should a patient have continued the interacting medication, most would have run out and been unable to obtain a refill within 90 days of receiving the letter, as this is the maximum amount dispensed at one time. The mean time to the first laboratory result for both gemfibrozil and niacin was 6.5 and 5.3 months, respectively. 
Approximately $85 \%$ of patients completed the first laboratory test at least 3 months after the letter was mailed.

The protocols were designed to assess whether gemfibrozil or niacin was indicated and did not assess whether the statin was indicated. Therefore, discontinuing the statin also could have resolved the interaction appropriately. However, due to characteristics of the patient population and recommendations in current lipid guidelines, it was more likely the statin would be indicated. ${ }^{22,23}$ The protocols also assumed that patients eligible for gemfibrozil or niacin discontinuation would not need additional changes to their lipid medications. The medication changes made by the PACT CPS may have gone beyond those minimally necessary to resolve the drug interaction and maintain TG goals. Patients who had gemfibrozil or niacin discontinued by protocol also may have benefited from additional optimization of their lipid medications.

\section{CONCLUSIONS}

This quality improvement analysis supports further evaluation of the complementary use of protocols and PACT CPS prescriptive authority to resolve statin drug interactions. The gemfibrozil and niacin protocols appropriately identified patients who were less likely to experience an adverse change in TG laboratory results. Patients more likely to require additional medication interventions were appropriately referred to the PACT Pharmacy Clinics for individualized care. These data support expanded roles for pharmacists, across various settings, to mitigate select drug interactions at the Truman VA.

\section{Acknowledgments}

This quality improvement project is the result of work supported with resources and use of the Harry S. Truman Memorial Veterans' Hospital in Columbia, Missouri.

\section{Author disclosures}

The authors report no actual or potential conflicts of interest with regard to this article.

\section{Disclaimer}

The opinions expressed herein are those of the authors and do not necessarily reflect those of Federal Practitioner, Frontline Medical Communications Inc., the US Government, or any of its agencies. This article may discuss unlabeled or investigational use of certain drugs. Please review the complete prescribing information for specific drugs or drug combinations -including indications, contraindications, warnings, and adverse effects-before administering pharmacologic therapy to patients.

\section{References}

1. The top 200 drugs of 2020 Provided by the ClinCalc DrugStats Database. http://clincalc.com/DrugStats /Top200Drugs.aspx. Updated February 11, 2017. Accessed May 12, 2020.

2. Wiggins BS, Saseen JJ, Page RL 2nd, et al; American Heart Association Clinical Pharmacology Committee of the Council on Clinical Cardiology; Council on Hypertension; Council on Quality of Care and Outcomes Research; and Council on Functional Genomics and Translational Biology. Recommendations for management of clinically significant drug-drug interactions with statins and select agents used in patients with cardiovascular disease: a scientific statement from the American Heart Association. Circulation. 2016;134(21):e468-e495. doi:10.1161/CIR.0000000000000456

3. Smithburger PL, Buckley MS, Bejian S, Burenheide K, Kane-Gill SL. A critical evaluation of clinical decision support for the detection of drug-drug interactions. Expert Opin Drug Saf. 2011;10(6):871-882. doi:10.1517/14740338.2011.583916

4. US Food and Drug Administration. FDA drug safety communication: new restrictions, contraindications, and dose limitations for Zocor (simvastatin) to reduce the risk of muscle injury. https://www.fda.gov/Drugs/DrugSafety /ucm256581.htm. Updated December 15, 2017. Accessed May 12, 2020.

5. US Food and Drug Administration. FDA drug safety communication: important safety label changes to cholesterol-lowering statin drugs. https://www.fda.gov /Drugs/DrugSafety/ucm293101.htm. Updated January 19, 2016. Accessed May 12, 2020.

6. US Food and Drug Administration Federal Register. AbbVie Inc. et al; withdrawal of approval of indications related to the coadministration with statins in applications for niacin extended-release tablets and fenofibric acid delayed-release capsules. https://www.federalregister .gov/documents/2016/04/18/2016-08887/abbvie-inc -et-al-withdrawal-of-approval-of-indications-related -to-the-coadministration-with-statins. Published April 18 2016. Accessed May 12, 2020.

7. Lamprecht DG Jr, Todd BA, Denham AM, Ruppe LK, Stadler SL. Clinical pharmacist patient-safety initiative to reduce against-label prescribing of statins with cyclosporine. Ann Pharmacother. 2017;51(2):140-145. doi:10.1177/1060028016675352

8. Roblek T, Deticek A, Leskovar B, et al. Clinical-pharmacist intervention reduces clinically relevant drugdrug interactions in patients with heart failure: $A$ randomized, double-blind, controlled trial. Int $J$ Cardiol. 2016;203:647-652. doi:10.1016/j.ijcard.2015.10.206

9. Tuchscherer RM, Nair K, Ghushchyan V, Saseen JJ. Simvastatin prescribing patterns before and after FDA dosing restrictions: a retrospective analysis of a large healthcare claims database. Am J Cardiovasc Drugs. 2015;15(1):27-34. doi:10.1007/s40256-014-0096-x

10. Alford JC, Saseen JJ, Allen RR, Nair KV. Persistent use of against-label statin-fibrate combinations from 2003-2009 despite United States Food and Drug Administration dose restrictions. Pharmacotherapy. 2012;32(7):623-630. doi:10.1002/j.1875-9114.2011.01090.x

11. Leape LL, Cullen DJ, Clapp MD, et al. Pharmacist participation on physician rounds and adverse drug events in the intensive care unit [published correction appears in JAMA 2000 Mar 8;283(10):1293]. JAMA. 1999;282(3):267-270. doi:10.1001/jama.282.3.267

12. Kucukarslan SN, Peters M, Mlynarek M, Nafziger DA. Pharmacists on rounding teams reduce preventable adverse drug events in hospital general medicine units. Arch Intern Med. 2003;163(17):2014-2018. 
doi:10.1001/archinte.163.17.2014

13. Humphries TL, Carroll N, Chester EA, Magid D, Rocho B. Evaluation of an electronic critical drug interaction program coupled with active pharmacist intervention. Ann Pharmacother. 2007;41(12):1979-1985. doi:10.1345/aph.1K349

14. Zocor [package insert]. Whitehouse Station, NJ: Merck \& Co, Inc; 2018

15. Lipitor [package insert]. New York, NY: Pfizer; 2017.

16. Crestor [package insert]. Wilmington, DE: AstraZeneca; 2018.

17. Mevacor [package insert]. Whitehouse Station, NJ: Merck \& Co, Inc; 2012.

18. Wolters Kluwer Health, Lexi-Drugs, Lexicomp. Pravastatin. www.online.lexi.com. [Source not verified.]

19. Miller M, Stone NJ, Ballantyne C, et al; American Heart Association Clinical Lipidology, Thrombosis, and Prevention Committee of the Council on Nutrition, Physical Activity, and Metabolism; Council on Arteriosclerosis, Thrombosis and Vascular Biology; Council on Cardiovascular Nursing; Council on the Kidney in Cardiovascular Disease. Triglycerides and cardiovascular disease: a scientific statement from the American Heart Association. Circulation. 2011;123(20):2292-2333. doi: 10.1161/CIR.0b013e3182160726

20. Ferguson J, Seston L, Ashcroft DM. Refer-to-pharmacy: a qualitative study exploring the implementation of an electronic transfer of care initiative to improve medicines optimisation following hospital discharge. BMC Health Serv Res. 2018;18(1):424.

doi:10.1186/s12913-018-3262-z

21. Ensing HT, Koster ES, Dubero DJ, van Dooren AA, Bouvy ML. Collaboration between hospital and community pharmacists to address drug-related problems: the HomeCoMe-program. Res Social Adm Pharm. 2019;15(3):267-278. doi:10.1016/j.sapharm.2018.05.001

22. US Department of Defense, US Department of Veterans Affairs. VA/DoD clinical practice guideline for the management of dyslipidemia for cardiovascular risk reduction guideline summary. https://www.healthquality.va.gov /guidelines/CD/lipids/LipidSumOptSinglePg31Aug15.pdf. Published 2014. Accessed May 14, 2020.

23. Stone NJ, Robinson JG, Lichtenstein AH, et al. 2013 ACC/AHA guideline on the treatment of blood cholesterol to reduce atherosclerotic cardiovascular risk in adults: a report of the American College of Cardiology/American Heart Association Task Force on Practice Guidelines [published correction appears in Circulation. 2014 Jun 24;129(25) (suppl 2):S46-48] [published correction appears in Circulation. $2015 \mathrm{Dec}$ 22;132(25):e396]. Circulation. 2014;129(25)(suppl 2): S1-S45. doi:10.1161/01.cir.0000437738.63853.7a 